\title{
Erratum to: Methylenetetrahydrofolate reductase (MTHFR) and thymidylate synthase (TS) polymorphisms with osteoporotic vertebral compression fracture (OVCF) in postmenopausal Korean women
}

\author{
Young Sun Chung - Young Joo Jeon - Dong Eun Shin · Kyung Tae Min - Yu Shik Shin • \\ Keun Soo Won - Young Cho Koh - Seung Ho Hong · Nam Keun Kim
}

(C) The Genetics Society of Korea and Springer 2012

Erratum to: Genes Genom (2012) 34: 257-263

DOI $10.1007 / \mathbf{s} 13258-011-0202-2$

The original version of this article contained an error in the Acknowledgements.

1. The Acknowledgements should be corrected as follow. Acknowledgements This work was supported by Konkuk University in 2012.

The online version of the original article can be found under doi:10.1007/s13258-012-0501-2

Y. S. Chung - Y. S. Shin · K. S. Won · Y. C. Koh

Department of Neurosurgery, KonKuk University School of Medicine, Korea

\section{Y. J. Jeon $\cdot$ K. T. Min · N. K. Kim $(\bowtie)$}

Institute for Clinical Research, CHA Bundang Medical Center, School of Medicine, CHA University, Seongnam, Korea

e-mail: nkkim@cha.ac.kr

D. E. Shin

Department of Orthopedic Surgery, CHA Bundang Medical Center, School of Medicine, CHA University, Seongnam, Korea

S. H. Hong $(\bowtie)$

Department of Science Education, Teachers College, Jeju National University, Jeju, Korea

e-mail: shong@jejunu.ac.kr 